\title{
A field theoretical approach to the Debye-Hückel electrolyte differential capacitance in a slab
}

\author{
D. di Caprio ${ }^{1}$, J.Stafiej ${ }^{2}$
}

1 Laboratoire d'Électrochimie et Chimie Analytique, UMR 7575, Université P. et M. Curie, Paris VI, Bât. F74, B.P. 39

4, Place Jussieu, 75230 Paris Cedex 05, France

2 Department of Electrode Processes, Institute of Physical Chemistry of the Polish Academy of Sciences, ul. Kasprzaka 44/52, 01-224 Warsaw, Poland

\section{Received September 18, 2001}

The effective Hamiltonian of our field theoretical model yields the linearized Debye-Hückel theory in the bulk. Here we consider it for a confined geometry of a slab. The differential capacitance is computed using two ways. The first relates it to the charge-charge correlation function by calculating the response to charging of the slab walls. The other relates it to the fluctuation of charge in one half of the slab. Since the model is exactly soluble in terms of the diagonal representation we can verify the fact that the two ways are consistent with each other and with the Gouy-Chapman differential capacitance in the limit of a thick slab. The interest of this derivation is to relate a measurable quantity to the structural information contained in the charge-charge correlation function.

Key words: ionic solutions, confined systems, correlation functions, Debye-Hückel electrolyte, statistical field theory, differential capacitance

PACS: $61.20 . \mathrm{Qg}, 73.30 .+y, 03.50 . \mathrm{Kk}$

\section{Introduction}

As this paper comes naturally out of our long work with Jean-Pierre Badiali we are very pleased to dedicate it to him on the occasion of his 60th anniversary.

The structure and the properties of inhomogeneous electrolyte solutions are important for interfacial electrochemistry, colloid and membrane sciences and therefore biological systems. A simple example of such an inhomogeneous system that we consider in our paper is an electrolyte solution bounded by two planar interfaces. Even if we focus on a single interface and consider a semi infinite system we have to deal with a complicated problem. Electrolyte solutions are multicomponent 
systems. They usually contain three species: two oppositely charged ions and the solvent. The components interact with each other and with the other side of the interface often reduced to a hard charged wall. These interactions can be both the long range coulombic interaction and the short range specific interactions. As a result of the complexity of these systems, people have been for many decades deeply attached to the simple physical description provided by the Gouy-Chapman theory. The physical ground of the theory is clear. It is a Mayer-McMillan level mean field theory based on the Poisson-Boltzmann equations for point like ions [1,2]. Also, it is a theory of reference in that it gives exact results, for instance on the differential capacitance for charged interfaces at a vanishing electric charge and at low ionic concentrations.

In a recent series of papers $[3,4]$ we have developed a field theoretical approach to study ionic solutions. We start from a simple Hamiltonian written in terms of fields describing the ionic distributions. The Hamiltonian includes the coulombic energy and the entropy. At the level of the quadratic approximation, this Hamiltonian yields the Debye-Hückel theory in the bulk. In this framework, we have calculated the charge-charge correlation function for a neutral interface [4]. We have shown that already in this simple case these correlations are quite complex, simply as a consequence of the presence of the hard bounding walls. In the case of charged interfaces, we have shown that this Hamiltonian gives in the mean field approximation the linearized Gouy-Chapman theory.

In this paper, we use our field theoretical approach to present two ways of deriving the differential capacitance for an ionic solution bounded by two hard plane walls. First, we consider the linear response regime and then we are able to calculate the response function to the charging of the walls from the knowledge of properties calculated for the neutral system. The differential capacitance is calculated from the charge-charge correlation function by means of the fluctuation dissipation theorem. Second, we divide the neutral system in two symmetric half systems and we focus on the charge fluctuations in any of the half systems. The aim of this paper is to show how the structural information available from the field theoretical approach is related to the differential capacitance which is a measurable quantity.

The paper is organized as follows. In section 2, we present the system and its effective Hamiltonian. For our inhomogeneous system, we describe briefly how we rewrite this Hamiltonian in a diagonal form to have a more tractable expression. The details of the calculations are given in [4]. In section 3, we describe the charge-charge correlation function in the neutral system at large wall separation. In section 4, we derive the differential capacitance in the linear response theory from the chargecharge correlation functions. We show that this result cannot be reduced to the physics included in the bulk correlation functions and we present the alternative derivation of the differential capacitance. Finally in section 5, we discuss what, in our opinion, the originality of the field theoretical approach is and suggest some guidelines for future investigations. 


\section{The model}

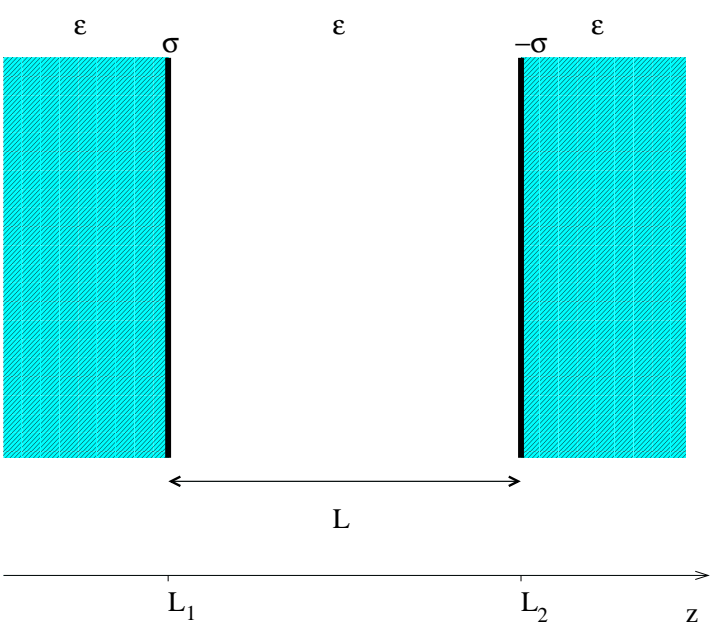

Figure 1. Schematic representation of the model system.

We consider a 1-1 ionic solution bounded by two hard walls at a distance $L$ from one another, as shown in figure 1 . The walls have a surface area $A$ and we will take the limit $A \rightarrow \infty$. The distance $L$ is finite and large in comparison with all other length scales in the system, e.g. the Debye length scale of the ionic solution or the average distance between ions. We consider a system of volume $A L$, with a fixed number $N_{+}, N_{-}$of ions. In the thermodynamic limit, the densities in the system $N_{+} /(A L), N_{-} /(A L)$ can be assimilated with the density found in a region in the middle of the system far from the two walls which will be referred to as the bulk. This defines a reference density $\rho_{b}$ which is the bulk density of anions and cations. The walls can be charged with a surface charge density $\sigma$. The dielectric constant is uniform throughout the whole space and corresponds to the dielectric constant of the pure solvent $\varepsilon$. The system is described in terms of fields $\rho_{+}(\mathbf{r}), \rho_{-}(\mathbf{r})$ which represent the ionic densities of the cations and of the anions respectively. To focus on the electrostatic effects, it is more convenient to introduce the equivalent representation: $q(\mathbf{r})=\rho_{+}(\mathbf{r})-\rho_{-}(\mathbf{r})$ and $s(\mathbf{r})=\rho_{+}(\mathbf{r})+\rho_{-}(\mathbf{r})$ in terms of charge density and overall ionic density. The Hamiltonian is a functional of the fields:

$$
\beta \mathcal{H}[q(\mathbf{r}), s(\mathbf{r})]=\beta \mathcal{H}^{\mathrm{ent}}[q(\mathbf{r}), s(\mathbf{r})]+\beta \mathcal{H}^{\operatorname{Coul}}[q(\mathbf{r})],
$$

where

$$
\beta \mathcal{H}^{\mathrm{Coul}}[q(\mathbf{r})]=\frac{K_{\mathrm{D}}^{2}}{8 \pi \rho_{b}} \int \frac{q(\mathbf{r}) q\left(\mathbf{r}^{\prime}\right)}{\tilde{r}} \mathrm{~d} \mathbf{r} \mathrm{d} \mathbf{r}^{\prime}+\beta e \int q(\mathbf{r}) V^{\text {ext }}(\mathbf{r}) \mathrm{d} \mathbf{r},
$$

$\tilde{r}=\left|\mathbf{r}-\mathbf{r}^{\prime}\right|, \beta=1 / k_{\mathrm{B}} T$ is the reciprocal temperature and $K_{\mathrm{D}}=\left(\beta \rho_{b} e^{2} / \varepsilon\right)^{1 / 2}$ is the inverse Debye length. $V^{\text {ext }}(\mathbf{r})$ is the potential created by the charges $\sigma$ and $-\sigma$ on the walls. This contribution is

$$
V^{\mathrm{ext}}(\mathbf{r})=-\frac{\sigma e}{\varepsilon}\left(z-\frac{L_{1}+L_{2}}{2}\right),
$$

where the zero for the electric potential corresponds to the middle of the system. For the entropy $\mathcal{H}^{\text {ent }}[q(\mathbf{r}), s(\mathbf{r})]$ we take the functional used in $[3,5]$ :

$$
\beta \mathcal{H}^{\mathrm{ent}}[q(\mathbf{r}), s(\mathbf{r})]=\int \sum_{i= \pm}\left[\rho_{i}(\mathbf{r}) \ln \frac{\rho_{i}(\mathbf{r})}{\rho}-1\right] \mathrm{d} \mathbf{r}
$$


and $\rho$ is an arbitrary reference scale for the entropy. Expanding around the bulk value of the charge density $q=0$ and of the total density $s=\rho_{b}$ and truncating to the quadratic form [3], we have

$$
\beta \mathcal{H}^{\mathrm{ent}}[q(\mathbf{r}), s(\mathbf{r})]=\beta \mathcal{H}^{\mathrm{ent}}\left[0, \rho_{b}\right]+\int \frac{q^{2}(\mathbf{r})+\left(s(\mathbf{r})-\rho_{b}\right)^{2}}{2 \rho_{b}} \mathrm{~d} \mathbf{r}+o\left(q^{2},\left(s-\rho_{b}\right)^{2}\right) .
$$

The functional integral over the fields yields the partition function

$$
Z[q, s]=\int \mathcal{D} q(\mathbf{r}) \mathcal{D} s(\mathbf{r}) \mathrm{e}^{-\beta \mathcal{H}[q(\mathbf{r}), s(\mathbf{r})]} .
$$

In the quadratic approximation there is no coupling between the $q$ and $s$ fields, as we focus on the excess properties related to the Coulomb interaction, we can formally integrate over the $s(\mathbf{r})$ degrees of freedom: $Z[q]=\int \mathcal{D} s(\mathbf{r}) Z[q, s]$. The partition function for the system is then $Z=\int \mathcal{D} q(\mathbf{r}) Z[q]$.

The interest of the bilinear model is that all the functional integrals can in principle be performed explicitly. To calculate the partition function or any average of a quantity in the system, we look for a diagonal representation of the Hamiltonian. The details of the calculation are given elsewhere [4] and we just sketch the steps of the calculation. First, we search for the eigenvectors of the operator potential

$$
V(\mathbf{r})=\frac{e}{4 \pi \varepsilon} \int \mathrm{d} \mathbf{r}^{\prime} \frac{q\left(\mathbf{r}^{\prime}\right)}{\left|\mathbf{r}-\mathbf{r}^{\prime}\right|}
$$

in the geometry of a system bounded by plane walls. Using the translational invariance in the direction parallel to the walls, we have shown in [4] that a suitable representation for the charge densities is

$$
q(\mathbf{r})=\sum_{\mathbf{K}} \mathrm{e}^{\mathrm{i} \mathbf{K R}} \sum_{\mu=\mu_{+}, \mu_{-}} \tilde{q}_{\mu}(\mathbf{K}) \phi_{\mu}(z),
$$

where the $\mu_{+}, \mu_{-}$verify respectively:

$$
\mathrm{e}^{\mathrm{i} \mu_{+} L}=\frac{K-\mathrm{i} \mu_{+}}{K+\mathrm{i} \mu_{+}} \quad \text { and } \quad \mathrm{e}^{\mathrm{i} \mu_{-} L}=-\frac{K-\mathrm{i} \mu_{-}}{K+\mathrm{i} \mu_{-}}
$$

where $L=L_{2}-L_{1}, K=|\mathbf{K}|$ and $\mu_{+}>0$ and $\mu_{-}>0$. The equations above yield the spectrum of $\mu_{+}$and $\mu_{-}$which depend on the value of $K$. The eigenfunctions are written:

$$
\phi_{\mu_{ \pm}}(z)=\mathcal{N}_{ \pm}\left[\mathrm{e}^{-\mathrm{i} \mu_{ \pm}\left(L_{2}-z\right)} \mp \mathrm{e}^{\mathrm{i} \mu_{ \pm}\left(L_{1}-z\right)}\right],
$$

where $\mathcal{N}_{ \pm}=\left[2 L\left(1 \mp \frac{\sin \left(\mu_{ \pm} L\right)}{\mu_{ \pm} L}\right)\right]^{-1 / 2}$ is the normalization constant and where the indices,+- refer to the type of eigenfunction. The eigenfunctions corresponding to $\mu_{+}$are odd while those corresponding to $\mu_{-}$are even with respect to the center at $z=\left(L_{1}+L_{2}\right) / 2$. The coefficients $\tilde{q}_{\mu}$ are complex valued. Changing the values of $\tilde{q}_{\mu}$, we explore all possible charge density distributions. The electric potential is then

$$
V(\mathbf{r})=\frac{e}{\varepsilon} \sum_{\mathbf{K}} \mathrm{e}^{\mathrm{i} \mathbf{K R}} \sum_{\mu=\mu_{+}, \mu_{-}} \lambda_{\mu} \tilde{q}_{\mu}(\mathbf{K}) \phi_{\mu}(z),
$$


where

$$
\lambda_{\mu}=\frac{1}{K^{2}+\mu^{2}}
$$

are the eigenvalues of the coulombic potential operator. We finally obtain the diagonal representation of our Hamiltonian :

$$
\beta \mathcal{H}[q]=\frac{A}{2 \rho_{b}} \sum_{\mathbf{K}, \mu>0} \tilde{q}_{\mu}(\mathbf{K}) \tilde{q}_{\mu}^{*}(\mathbf{K})\left[1+\frac{K_{\mathrm{D}}^{2}}{K^{2}+\mu^{2}}\right]
$$

\section{Charge-charge correlation function for the neutral system}

For neutral walls, we have obtained the charge-charge correlation functions [4] for the semi infinite system. Here we can use these expressions considering $L$ to be large with respect to any length scale. The charge-charge correlation function has two contributions: one dependent on $z-z^{\prime}$ is translationally invariant and yields the bulk correlation function [3]:

$$
\begin{aligned}
g_{q q}^{b}\left(\mathbf{r}, \mathbf{r}^{\prime}\right) & =\left\langle q(\mathbf{r}) q\left(\mathbf{r}^{\prime}\right)\right\rangle_{b} \\
& =\rho_{b}\left[\delta\left(\mathbf{r}-\mathbf{r}^{\prime}\right)-\frac{K_{\mathrm{D}}^{2}}{4 \pi} \frac{\mathrm{e}^{-K_{\mathrm{D}} \tilde{r}}}{\tilde{r}}\right]
\end{aligned}
$$

where $\delta\left(\mathbf{r}-\mathbf{r}^{\prime}\right)$ is the Dirac function in space.

The term dependent on $z+z^{\prime}$ is a function of the distance to the wall. For $\mathbf{r}$ and $\mathbf{r}^{\prime}$ inside the slab, the charge-charge correlation function is given by

$$
\begin{aligned}
g_{q q}^{s}\left(\mathbf{r}, \mathbf{r}^{\prime}\right)= & \left\langle q(\mathbf{r}) q\left(\mathbf{r}^{\prime}\right)\right\rangle_{s} \\
= & \frac{\rho_{b} \mathrm{e}^{-K_{\mathrm{D}} \tilde{r}_{1}}}{2 \pi}\left[\frac{K_{\mathrm{D}}^{2}}{2 \tilde{r}_{1}}+\frac{K_{\mathrm{D}}}{\tilde{r}_{1}^{2}}-\frac{K_{\mathrm{D}}^{2} \tilde{z}_{1}^{2}-1}{\tilde{r}_{1}^{3}}-3 \frac{K_{\mathrm{D}} \tilde{z}_{1}^{2}}{\tilde{r}_{1}^{4}}-3 \frac{\tilde{z}_{1}^{2}}{\tilde{r}_{1}^{5}}\right] \\
& +\frac{\rho_{b}}{2 \pi} \int K^{2} \mathrm{~d} K J_{0}\left(K\left|\mathbf{R}-\mathbf{R}^{\prime}\right|\right) \mathrm{e}^{-\tilde{z}_{1}} \sqrt{K^{2}+K_{\mathrm{D}}^{2}} \\
& + \text { same terms with } \tilde{z}_{1} \rightarrow \tilde{z}_{2} \text { and } \tilde{r}_{1} \rightarrow \tilde{r}_{2},
\end{aligned}
$$

where $\tilde{z}_{1}=L_{1}-z+L_{1}-z^{\prime}$ and $\tilde{z}_{2}=L_{2}-z+L_{2}-z^{\prime}$ and $J_{0}$ is a Bessel function [6]. $\tilde{r}_{1}$ is the distance between the point $\mathbf{r}$ and the image $\mathbf{r}_{1}^{\prime}$ of the point $\mathbf{r}^{\prime}$ symmetrically with respect to the wall situated at $L_{1}$, and $\tilde{r}_{2}$ the same with respect to the other wall in $L_{2}$. The charge-charge correlations close to the interfaces thus have a complex behaviour even for neutral interfaces. The first contribution in brackets is screened over the Debye length and behaves like an interaction with an image charge, although there is no image potential at the dividing wall itself. The last integral is discussed in [4] and has a peculiar long ranged $R^{-3}$ behaviour which has first been discussed by B.Jancovici [7]. 


\section{Differential capacitance from the fluctuation dissipation theorem}

To calculate the capacitance of the system we consider the linear response theory for the Hamiltonian for a small charge density $\sigma$. The Hamiltonian can be rewritten

$$
\begin{aligned}
\mathcal{H} & =\mathcal{H}(\sigma=0)+\mathcal{H}_{\sigma}, \\
\mathcal{H}_{\sigma} & =e \int q(\mathbf{r}) V^{\text {ext }}(\mathbf{r}) \mathrm{d} \mathbf{r} .
\end{aligned}
$$

The term proportional to $\sigma$ in the Hamiltonian is taken as a perturbation and all averages are then computed for $\sigma=0$. At the linear response approximation, the average of a quantity $\mathcal{Q}$ is then

$$
\langle\mathcal{Q}\rangle_{\sigma}=\left\langle\mathcal{Q}\left(1-\beta \mathcal{H}_{\sigma}\right)\right\rangle_{0},
$$

where $\langle\ldots\rangle_{\sigma}$ and $\langle\ldots\rangle_{0}$ denote respectively averages taken with the full Hamiltonian and the Hamiltonian for zero charge on the walls. From the translational invariance in the direction parallel to the walls, the electric potential is independent of the position of the point we choose inside a plane parallel to the walls and depends only on the variable $z$. The potential difference between the two walls can then be written in the following way:

$$
\begin{aligned}
V\left(L_{2}\right)-V\left(L_{1}\right)= & \left\langle\left(V^{\mathrm{ext}}(r)+\frac{e}{4 \pi \varepsilon} \int \frac{q\left(\mathbf{r}^{\prime}\right)}{\left|\mathbf{r}-\mathbf{r}^{\prime}\right|} \mathrm{d} \mathbf{r}^{\prime}\right)\right. \\
& \left.\times\left(1-\beta e \int q\left(\mathbf{r}^{\prime \prime}\right) V^{\mathrm{ext}}\left(\mathbf{r}^{\prime \prime}\right) \mathrm{d} \mathbf{r}^{\prime \prime}\right)\right\rangle_{0, z=L_{2}}-\langle\ldots\rangle_{0, z=L_{1}} \\
= & \delta V^{\mathrm{ext}}+\frac{e}{4 \pi \varepsilon}\left[\left\langle\int \frac{q\left(\mathbf{r}^{\prime}\right)}{\left|\mathbf{r}-\mathbf{r}^{\prime}\right|} \mathrm{d} \mathbf{r}^{\prime}\right\rangle_{0, z=L_{2}}-\langle\ldots\rangle_{0, z=L_{1}}\right] \\
& -\frac{\beta e^{2}}{4 \pi \varepsilon}\left[\left\langle\int \frac{q\left(\mathbf{r}^{\prime}\right)}{\left|\mathbf{r}-\mathbf{r}^{\prime}\right|} \mathrm{d} \mathbf{r}^{\prime} \int q\left(\mathbf{r}^{\prime \prime}\right) V^{\operatorname{ext}}\left(\mathbf{r}^{\prime \prime}\right) \mathrm{d} \mathbf{r}^{\prime \prime}\right\rangle_{0, z=L_{2}}-\langle\ldots\rangle_{0, z=L_{1}}\right] \\
& -\beta e\left[\left\langle V^{\operatorname{ext}}\left(L_{2}\right) \int q\left(\mathbf{r}^{\prime \prime}\right) V^{\operatorname{ext}}\left(\mathbf{r}^{\prime \prime}\right) \mathrm{d} \mathbf{r}^{\prime \prime}\right\rangle_{0, z=L_{2}}-\langle\ldots\rangle_{0, z=L_{1}}\right], \quad(19)
\end{aligned}
$$

where for each quantity its difference for $z=L_{2}$ and $z=L_{1}$ is taken. The first term is $\delta V^{\text {ext }}=V^{\text {ext }}\left(z=L_{2}\right)-V^{\text {ext }}\left(z=L_{1}\right)=-\sigma e L / \varepsilon$ the potential difference due to the electric charges on the wall. The second term is the average of the Coulomb potential created by the charges in the ionic solution but taken for neutral walls. In this case, the electric potential is constant throughout the system and this difference is zero [3]. The last term is proportional to $\sigma^{2}$ and can be neglected in the linear response regime.

We then have to compute:

$$
\begin{aligned}
\delta \tilde{V} & \equiv V\left(L_{2}\right)-V\left(L_{1}\right)-\delta V^{\mathrm{ext}} \\
& =-\frac{\beta e^{2}}{4 \pi \varepsilon}\left[\left.\left\langle\int \frac{q\left(\mathbf{r}^{\prime}\right)}{\left|\mathbf{r}-\mathbf{r}^{\prime}\right|} \mathrm{d} \mathbf{r}^{\prime} \int q\left(\mathbf{r}^{\prime \prime}\right) V^{\operatorname{ext}}\left(\mathbf{r}^{\prime \prime}\right) \mathrm{d} \mathbf{r}^{\prime \prime}\right\rangle_{0}\right|_{z=L_{2}}-\left.\langle\ldots\rangle_{0}\right|_{z=L_{1}}\right]
\end{aligned}
$$


where terms of the order $\sigma^{2}$ are neglected. In our representation we have

$$
\begin{aligned}
\int q(\mathbf{r}) V^{\mathrm{ext}}(\mathbf{r}) \mathrm{d} \mathbf{r} & =-\frac{\sigma e A}{\varepsilon} \int_{L_{1}}^{L_{2}} \mathrm{~d} z^{\prime} z^{\prime} \sum_{\mu=\mu_{ \pm, K=0}} \tilde{q}_{\mu_{ \pm}} \phi_{\mu_{ \pm}}\left(z^{\prime}\right) \\
& =-\frac{4 \sigma e A}{\varepsilon} \sum_{\mu=\mu_{+, K=0}} \mathcal{N}_{+} \frac{\tilde{q}_{\mu}}{\mu^{2}}
\end{aligned}
$$

where $\phi_{\mu_{ \pm}}\left(z^{\prime}\right)$ has been replaced by its expression from equation (10) and the external potential $V^{\text {ext }}$ depending on $z$ uniquely selects $\mathbf{K}=0$ wave vector when integrating over the direction parallel to the walls. In this case $\mathrm{e}^{\mathrm{i} \mu_{ \pm} L}=\mp 1$. Also, only the $\mu_{+}$eigenvalues give a non-vanishing contribution to the sum.

From the expression of the electric potential in our representation we deduce

$$
\left.\int \frac{q\left(\mathbf{r}^{\prime}\right)}{\left|\mathbf{r}-\mathbf{r}^{\prime}\right|} \mathrm{d} \mathbf{r}^{\prime}\right|_{z}=4 \pi \sum_{\mu=\mu_{ \pm}} \tilde{q}_{\mu} \frac{\phi_{\mu}(z)}{\mu^{2}+K^{2}}
$$

The average over $\tilde{q}_{\mu}$ is performed with the Hamiltonian given in equation (13). It is diagonal in this representation and therefore we select the $\mathbf{K}=0$ and $\mu=\mu_{+}$vector also for this term. Thus we have the difference

$$
\left.\int \frac{q\left(\mathbf{r}^{\prime}\right)}{\left|\mathbf{r}-\mathbf{r}^{\prime}\right|} \mathrm{d} \mathbf{r}^{\prime}\right|_{L_{2}}-\left.\int \frac{q\left(\mathbf{r}^{\prime}\right)}{\left|\mathbf{r}-\mathbf{r}^{\prime}\right|} \mathrm{d} \mathbf{r}^{\prime}\right|_{L_{1}}=4 \pi \sum_{\mu_{+, K=0}} \tilde{q}_{\mu_{+}} \frac{4 \mathcal{N}_{+}}{\mu_{+}^{2}},
$$

where we have used that for $\mathbf{K}=0, \phi_{\mu_{+}}\left(L_{2}\right)-\phi_{\mu_{+}}\left(L_{1}\right)=4 \mathcal{N}_{+}$. Finally the potential difference is

$$
\delta \tilde{V}=\frac{\beta e^{3} \sigma A}{\varepsilon^{2}} \sum_{\mu_{+, K=0}} \frac{16 \mathcal{N}_{+}^{2}}{\mu_{+}^{4}}\left\langle\tilde{q}_{\mu_{+}} \tilde{q}_{\mu_{+}}^{*}\right\rangle_{0} .
$$

From the Hamiltonian we see that the average $\left\langle\tilde{q}_{\mu_{+}} \tilde{q}_{\mu_{+}}^{*}\right\rangle_{0}=\rho_{b} \mu_{+}^{2} /\left[A\left(\mu_{+}^{2}+K_{\mathrm{D}}^{2}\right)\right]$; thus,

$$
\delta \tilde{V}=\frac{8 \sigma e}{\varepsilon L} \sum_{\mu_{+, K=0}}\left[\frac{1}{\mu_{+}^{2}}-\frac{1}{\mu_{+}^{2}+K_{\mathrm{D}}^{2}}\right] .
$$

The calculation of the sums over $\mu_{+}$is presented in the appendix

$$
\sum_{\mu_{+}, K=0} \frac{1}{\mu_{+}^{2}}=\frac{L^{2}}{8} \quad \text { and } \quad \sum_{\mu_{+, K=0}} \frac{1}{\mu_{+}^{2}+K_{\mathrm{D}}^{2}}=\frac{L}{4 K_{\mathrm{D}}}\left[1-\mathrm{e}^{-K_{\mathrm{D}} L}\right] .
$$

Finally, we have

$$
\begin{aligned}
V\left(L_{2}\right)-V\left(L_{1}\right) & =\delta V^{\mathrm{ext}}+\frac{\sigma e L}{\varepsilon}-\frac{2 \sigma e}{\varepsilon K_{\mathrm{D}}}\left[1-\mathrm{e}^{-K_{\mathrm{D}} L}\right] \\
& =-\frac{2 \sigma e}{\varepsilon K_{\mathrm{D}}}\left[1-\mathrm{e}^{-K_{\mathrm{D}} L}\right] .
\end{aligned}
$$

We then obtain the capacitance deriving $V\left(L_{2}\right)-V\left(L_{1}\right)$ with respect to $-\sigma e$ (the minus is due to the conventions chosen in figure 1$)$. We find that the first sum in 
equation (27) compensates the external potential $\delta V^{\text {ext }}$. The differential capacitance per unit of surface area at the point of zero charge is obtained

$$
\begin{aligned}
C^{-1} & =\frac{\tilde{V}\left(L_{2}\right)-\tilde{V}\left(L_{1}\right)}{-\sigma e} \\
& =\frac{2}{\varepsilon K_{\mathrm{D}}}\left[1-\mathrm{e}^{-K_{\mathrm{D}} L}\right]
\end{aligned}
$$

which in the large $L$ limit corresponds to the differential capacitance obtained in the linearized Gouy-Chapman theory.

We believe that the interest in deriving a well known result in a different way resides in trying to give it a new interpretation. The differential capacitance has been obtained from the theorem of fluctuation dissipation, let us rewrite equation (19) so that the charge-charge correlation function appears explicitly

$$
\begin{aligned}
\delta \tilde{V}=-\frac{\beta e^{2}}{4 \pi \varepsilon A}[ & \left.\int \frac{\left\langle q\left(\mathbf{r}^{\prime}\right) q\left(\mathbf{r}^{\prime \prime}\right)\right\rangle_{0}}{\left|\mathbf{r}-\mathbf{r}^{\prime}\right|}\right|_{z=L_{2}} V^{\mathrm{ext}}\left(\mathbf{r}^{\prime \prime}\right) \mathrm{d} \mathbf{r}^{\prime \prime} \mathrm{d} \mathbf{r}^{\prime} d \mathbf{R} \\
& \left.- \text { same term for } z=L_{1}\right] .
\end{aligned}
$$

Note that due to the translational invariance parallel to the wall, the integration over $\mathbf{R}$ amounts to introducing the surface $A$ as a factor. As for the neutral system, the charge profile is zero and the ionic profiles are uniform, one might suppose that the Debye Hückel bulk correlation function is sufficient to calculate the linear response function. If we replaced $\left\langle q\left(\mathbf{r}^{\prime}\right) q(\mathbf{r})\right\rangle_{0}$ by the charge-charge correlation function given in equation (14), we would have

$$
\begin{aligned}
\delta \tilde{V}=-\frac{\beta e^{2}}{4 \pi \varepsilon A} \int & \rho_{b}\left[\delta\left(\mathbf{r}^{\prime}-\mathbf{r}^{\prime \prime}\right)-\frac{K_{\mathrm{D}}^{2}}{4 \pi} \frac{e^{-K_{\mathrm{D}}\left|\mathbf{r}^{\prime}-\mathbf{r}^{\prime \prime}\right|}}{\mathbf{r}^{\prime}-\mathbf{r}^{\prime \prime}}\right]\left(-\frac{\sigma e}{\varepsilon}\right)\left[z^{\prime \prime}-\left(\frac{L_{1}+L_{2}}{2}\right)\right] \\
& \times 4 \pi\left[z^{\prime}-\left(\frac{L_{1}+L_{2}}{2}\right)\right] \mathrm{d} \mathbf{r}^{\prime} \mathrm{d} \mathbf{r}^{\prime \prime}
\end{aligned}
$$

We have used the fact that the integral over $\mathbf{R}$ is the same as the integral expressing the electric potential created by a charged plane $z=L_{2}$ at the point $\mathbf{r}^{\prime}$. Performing all the integrations we find

$$
\delta \tilde{V}=-\frac{2 \sigma e}{\varepsilon K_{\mathrm{D}}}+2\left(\frac{K_{\mathrm{D}} L}{2}\right)^{2} \frac{\sigma e}{\varepsilon K_{\mathrm{D}}} .
$$

The differential capacity is then

$$
C^{-1}=\frac{2 e}{\varepsilon K_{\mathrm{D}}}-2\left(\frac{K_{\mathrm{D}} L}{2}\right)^{2} \frac{1}{\varepsilon K_{\mathrm{D}}}+\frac{L}{\varepsilon} .
$$

We readily see that this is not the correct result. In particular, there is a macroscopic term in $L^{2}$ which is not compensated and there is no linear term which should cancel the external electric potential created by the charges on the wall. This shows that 
the proper form of the charge-charge correlation is required to obtain the correct differential capacitance.

We have seen that only the odd modes contribute to the differential capacitance given in equation (32). For the system in an odd mode there is a non-vanishing charge in the half space $z<\left(L_{1}+L_{2}\right) / 2$ compensated by the charge in the other half space. This suggests that charge transfer between the two halves of the system is important. The charge in one half of the system is

$$
Q=\int_{L_{1}}^{\frac{L_{1}+L_{2}}{2}} q(\mathbf{r}) \mathrm{d} \mathbf{r}=-\int_{\frac{L_{1}+L_{2}}{2}}^{L_{2}} q(\mathbf{r}) \mathrm{d} \mathbf{r} .
$$

The fluctuation of the charge of a system in contact with a reservoir is related with its differential capacitance $\tilde{C}[10]$

$$
\left\langle Q^{2}\right\rangle=A \tilde{C} k_{\mathrm{B}} T
$$

If we can treat the other half of the system as a charge reservoir we can calculate $\left\langle Q^{2}\right\rangle$ using the diagonal representation.

$$
\begin{aligned}
\left\langle Q^{2}\right\rangle & =\rho_{b} e^{2} A \sum_{\mu>0} \frac{\mu^{2}}{\mu^{2}+K_{\mathrm{D}}^{2}}\left|\int_{L_{1}}^{\frac{L_{1}+L_{2}}{2}} \phi_{\mu}(z) \mathrm{d} z\right|^{2} \\
& =A k_{\mathrm{B}} T \varepsilon K_{\mathrm{D}}\left(1-\mathrm{e}^{-K_{\mathrm{D}} L}\right) .
\end{aligned}
$$

As before, this sum contains the contribution from the odd modes only. In the large $L$ limit we retrieve the linearized Gouy-Chapman differential capacitance. For finite $L$ we can note that the differential capacitance in equation (32) and equation (39) is not the same. In particular for $L \rightarrow 0$ the limits are completely different showing that the other half system cannot be treated as a charge reservoir in this limit.

\section{Conclusions}

In this paper we have reobtained a well established quantity - the Gouy-Chapman differential capacity, which is an important measurable characteristic of the inhomogeneous electrolyte system. We use a field theoretical framework for handling the problem of the long range Coulomb interactions. In this framework the ionic densities play the role of the fields. The interest of this is that the fields have got a clear physical interpretation. The charge-charge correlations appear naturally with such a choice of the fields in our formalism. We can relate them to the differential capacitance. Our field theoretical effective Hamiltonian provides a description equivalent to the linearized Debye-Hückel theory in the bulk solution, which is exact in the low density limit [3]. As we have seen, this Hamiltonian gives a tractable model for the slab geometry. Due to this we have been able to verify the validity of some assumptions. Even in the limit of vanishing charge on the walls, the bulk correlation function is not sufficient to approximate the inhomogeneous correlation function. Only in the limit of a thick slab the differential capacitance calculated from 
the response to an external charge is consistent with the capacitance calculated from the charge fluctuation in the half system. Otherwise we see the effect of the finite size on the calculated differential capacitance which makes the difference between the two results. This sheds some light on the problem of the choice of the electric variable to describe the interfacial systems discussed in electrochemistry [11].

Here we have focused on the simplest and the best understood case of the linearized Debye Hückel electrolyte model. In the future we intend to go beyond the linear model described by the bilinear Hamiltonian and/or introduce specific interactions. A great simplification comes from the fact that these interactions are introduced in the spirit of the field theory and not at the molecular level.

\section{Acknowledgements}

We gratefully acknowledge the joined financial support by the National Committee for Scientific Research (KBN) of Poland and the Ministry of Research in France, within the agreement Polonium project $\mathrm{N}^{\circ}$ 01589ZE.

\section{Appendix}

We calculate the sums $\sum_{f_{+}(\mu)=0, \mu>0} 1 / \mu^{2}$ and $\sum_{f_{+}(\mu)=0, \mu>0} 1 /\left(\mu^{2}+K_{\mathrm{D}}^{2}\right)$ where $f_{+}(\mu)=\mathrm{e}^{\mathrm{i} \mu L}+1$, which corresponds to $\mu_{+}=\pi / L+n[2 \pi / L]$ where $\mathrm{n}$ is an integer. The first sum is simply [9]

$$
\begin{aligned}
\sum_{f_{+}(\mu)=0, \mu>0} \frac{1}{\mu^{2}} & =\frac{L^{2}}{\pi^{2}} \sum_{n=0}^{\infty} \frac{1}{(2 n+1)^{2}} \\
& =\frac{L^{2}}{8} .
\end{aligned}
$$

For the second sum, we use the following identity

$$
\sum_{f_{+}(\mu)=0} \phi(\mu)=\frac{1}{2 \pi \mathrm{i}} \oint_{\mathcal{C}} \frac{f_{+}^{\prime}(\mu)}{f_{+}(\mu)} \phi(\mu) \mathrm{d} \mu
$$

where the ' denotes the derivative with respect to $\mu$ and where $\phi(\mu)$ is an analytic function inside the contour integral $\mathcal{C}$ and extend over all values of $\mu$ positive and negative. In our case

$$
\frac{f_{+}^{\prime}(\mu)}{f_{+}(\mu)}=\frac{\mathrm{i} L \mathrm{e}^{\mathrm{i} \mu L}}{\mathrm{e}^{\mathrm{i} \mu L}+1} \quad \text { and } \quad \phi(\mu)=\frac{1}{\mu^{2}+K_{\mathrm{D}}^{2}} .
$$

The contour $\mathcal{C}$, shown in figure 2 , is chosen in order to include all the zeros of $f(\mu)$ which are on the real axis, in the limit $\delta_{1} \rightarrow \infty$ and $\delta_{2} \rightarrow 0$. It is possible to check that the portion of the contour parallel to the imaginary axis gives a vanishing contribution with $\delta_{2}$. The other two contributions to the contour denoted $I_{1}$ and $I_{2}$ can be computed using two other contours shown in figure 3 , these contours are also 


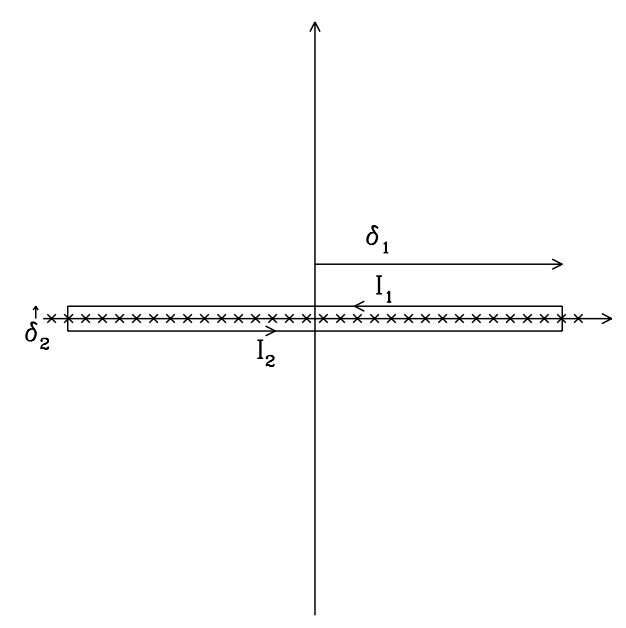

Figure 2. Contour in the complex plane used to calculate the sums in the Appendix. The poles $\mu_{+}$are indicated by the crosses on the real axis.

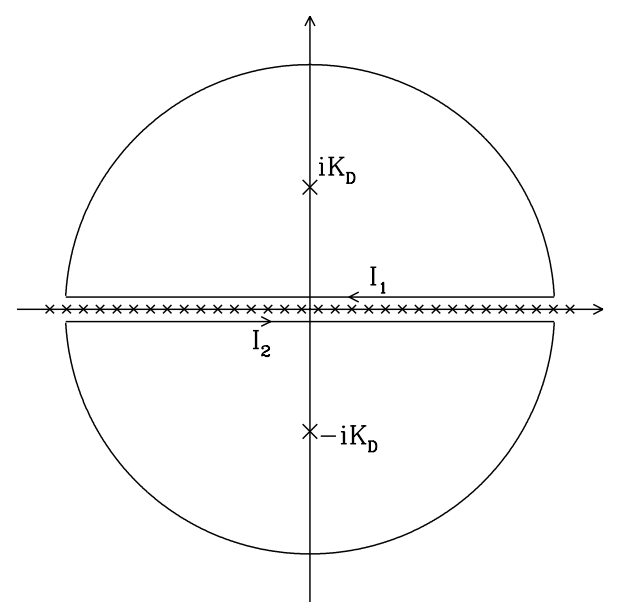

Figure 3. Contour in the complex plane used to calculate the intermediate integrals $I_{1}$ and $I_{2}$ in the Appendix.

considered in the limit $\delta_{1} \rightarrow \infty$. The arc of circles does not contribute to the integral in this limit, the integrals $I_{1}$ and $I_{2}$ are then residue of the poles respectively $\mathrm{i} K_{\mathrm{D}}$ and $-\mathrm{i} K_{\mathrm{D}}$ and we obtain:

$$
I_{1}=\frac{-L \mathrm{e}^{-K_{\mathrm{D}} L}}{2 K_{\mathrm{D}}}
$$

and

$$
I_{2}=\frac{L}{2 K_{\mathrm{D}}}
$$

thus, the sum we want to calculate is

$$
\sum_{f_{+}(\mu)=0, \mu>0} \frac{1}{\mu^{2}+K_{\mathrm{D}}^{2}}=\frac{L}{4 K_{\mathrm{D}}}\left[1-\mathrm{e}^{-K_{\mathrm{D}} L}\right] .
$$

Note that the contribution $I_{1}$ is negligible compared with $I_{2}$, since $L$ is much larger than $K_{\mathrm{D}}^{-1}$.

\section{References}

1. McMillan W.G., Mayer I.J.E. // J. Chem. Phys., 1945, vol. 13, p. 276.

2. Spaarnay M.J. The Electrical Double Layer. - In: The International Encyclopedia of Physical Chemistry And Chemical Physics. Oxford, Pergamon Press, 1972.

3. Stafiej J., Badiali J.P. // J. Chem. Phys., 1997, vol. 106, p. 8579-8586; D. di Caprio, Stafiej J., Badiali J.P. // J. Chem. Phys., 1998, vol. 108, p. 8572-8583.

4. di Caprio D., Stafiej J., Badiali J.P., Borkowska Z. // Electrochimica Acta, vol. 46, No. 20-21, p. 3051-3055. 
5. Frusawa H., Hayakawa R. // Phys. Rev. E, 1999, vol. 60, p. R5048-R5051.

6. Spanier J., Oldham K.B. An Atlas of Functions. Washington, Hemisphere Publishing Corporation, 1987; Abramowitz M., Stegun I.A. Handbook of Mathematical Functions with Formulas, Graphs and Mathematical Tables. - Applied Mathematics Series 55. Washington, National Bureau of Standards, 1965.

7. Jancovici B. // J. Stat. Phys., 1982, vol. 28, p. 43; J. Stat. Phys., 1982, vol. 29, p. 263; J. Stat. Phys., 1995, vol. 80, p. 445.

8. Stafiej J., di Caprio D., Badiali J.P. // Phys. Rev. E, 2000, vol. 61, p. 3877-3883.

9. Gradshteyn I.S., Ryzhik I.M. Table of Integrals, Series, and Products. (Edited by Alan Jeffrey) 5th edition, San Diego, Academic Press, 1994.

10. Mc Combie - In: Problems in Thermodynamics and Statistical Physics. Ed. Landsberg P.T., London, Pion, 1971, p. 457-460.

11. Partenskii M.B., Dorman V.L., Jordan P.C. // Int. Revs. Phys. Chem., 1996, vol. 15, p. $153-182$.

12. Evans R. // Adv. Phys., 1979, vol. 28, p. 143.

\title{
Теоретико-польовий підхід до диференційної ємності електроліта Дебая-Гюкеля в пластині
}

\author{
Д. ді Капріо ${ }^{1}$, Я.Стафє ${ }^{2}$ \\ 1 Лабораторія електрохімії та аналітичної хімії, УМР 7575, \\ Університет П. і М.Кюрі, 75230 Париж, Франція \\ 2 Інститут фізичної хімії Академії наук Польщі, Варшава, Польща
}

Отримано 18 вересня 2001 р.

Ефективний гамільтоніан нашої теоретико-польової моделі дає лінеаризовану теорію Дебая-Гюкеля для об'ємних властивостей. Тут ми розглядаємо його для обмеженої геометрії пластини. Диференціальна ємність обчислюється двома способами. Перший пов'язує їі з прямою кореляційною функцією заряд-заряд на основі обчислення відгуку до заряджання стінок пластини. Інші методи пов'язують її з флуктуацією заряду в одній половині пластини. Оскільки модель $€$ точно розв'язувана в термінах діагонального представлення, ми можемо перевірити той факт, що два способи узгоджуються між собою і з виразом для диференційної ємності Гуї-Чепмена в границі тонкої пластини. Цінність цього висновку мусить пов'язувати вимірювальну величину зі структурною інформацією, яка міститься в кореляційній функції заряд-заряд.

Ключові слова: іонні розчини, обмежені системи, кореляційні функції, електроліт Дебая-Гюкеля, статистико-польова теорія, диференційна ємність

PACS: $61.20 . \mathrm{Qg}, 73.30 .+y, 03.50 . \mathrm{Kk}$ 